\title{
Solid-phase microextraction: a multi-purpose microtechnique
}

\author{
"Biological matrices are complex but stable and reproducible; thus, external \\ calibration can be successfully used, facilitating accurate and precise measurements. In \\ addition, kinetic calibration (i.e., standard on fiber) is accessible for short sampling."
}

Keywords: automation $₫$ clinical applications $\approx$ environmental analysis $₫$ food studies $\approx$ high throughput " metabolomics $=$ on-site analysis $\|$ solid-phase microextraction

The growing number of measurements involving living systems influences the development of new analytical methods and instrumentation. There is no 'magic bullet' that will cover all the needs and expectations of researchers, but the introduction of novel techniques may open new possibilities and cover some gaps in current analytical approaches. Herein, we summarize the ways in which solid-phase microextraction (SPME) can complement currently used techniques.

\section{Milestones in SPME}

Since the invention of SPME in the early 1990s, its significant development has allowed its use to spread to different disciplines. New directions were induced by current needs and trends in analytical chemistry. The first SPME device was an optical fiber covered with a layer of polymeric material, which eventually developed into a metal wire or blade coated with a biocompatible polymer. In order to improve the feasibility of SPME in daily analysis, and to allow its coupling with common analytical instruments such as GC, a microsyringe-type device, housing the SPME fiber with a fused silica or stainless steel rod, was constructed. In parallel with the development of this SPME syringe and the constant evaluation of new coatings, the need for high-throughput analysis has led to the introduction of automated SPME systems for GC analysis. Straightforward coupling of SPME devices with GC (GC-MS) platforms led to the wide use of SPME in fragrance analysis, environmental research and headspace food studies [1]. By implementing coatings applicable for direct immersion extraction, SPME expanded to the measurement of nonvolatile and thermally labile compounds using a LC method, initially via manual extraction and finally in automated mode. Additionally, the improvement of SPME-LC interfaces has led to an increased number of applications analyzing non-volatile compounds. Direct extraction from complex matrices, for example, biofluids, became possible with the development of sorbents, which are covered with a thin layer of biocompatible polymer to protect the adhesion of large molecules, such as proteins or phospholipids, and provide good sample clean-up. This facilitated the application of SPME analysis in the biomedical field and its successful use in clinical practice. The most promising application of SPME, which distinguishes this method from other extraction and sample preparation techniques, is its applicability to in vivo and on-site sampling. In this case, SPME combines sampling, sample preparation and extraction in one step. Biological matrices are complex but stable and reproducible; thus, external calibration can be successfully used, facilitating accurate and precise measurements. In addition, kinetic calibration (i.e., standard on fiber) is accessible for short sampling [2]. Moreover, in vivo SPME allows the analysis of unstable and short-lived compounds, as will be discussed later in this article.

\section{Recent trends in SPME}

- Automation \& high throughput

Since its invention, several implementations of SPME (e.g., fiber, vessel, stirrer, suspended particles, disk/membrane and in-tube) have been considered [3]. Among the available geometries, coated fiber is the most common form of SPME, in conjunction with both GC and LC systems. In order to improve sample preparation strategies for fast analysis, automation and parallel analysis have been studied over the years. Automation simplifies demanding and time-consuming sample

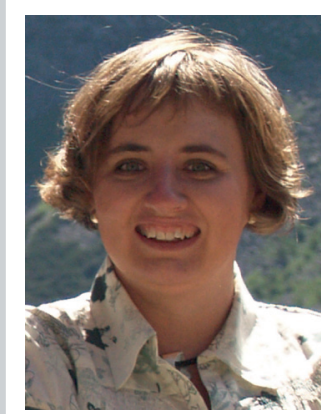

Barbara Bojko

Department of Chemistry, University of Waterloo, Canada

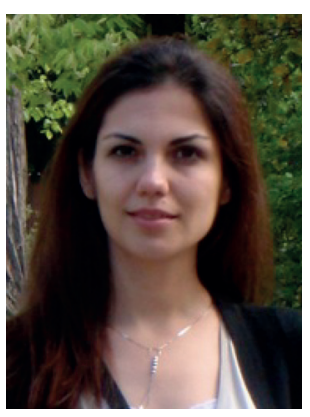

Fatemeh Mirnaghi

Department of Chemistry, University of Waterloo, Canada

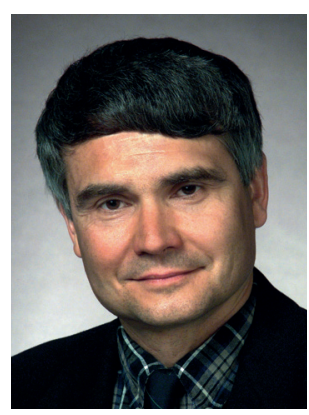

Janusz Pawliszyn

Author for correspondence: Department of Chemistry, University of Waterloo, Ontario, N2L 3GI, Canada

Tel.: + I 519888464 |

Fax: + I 5197460435

E-mail: janusz@sciborg.uwaterloo.ca 
preparation steps and improves the accuracy of the analysis. Sampling rate and throughput can be improved by analyzing several samples simultaneously. Several commercial robotic SPME autosamplers have been developed for automated extraction and desorption of analytes, where many food, environmental and biological applications of automated SPME have been reported. In the first high-throughput 96-multiwell SPME-LC application, a commercially available pin-tool replicator was used to support 96 pins, onto which hollow polydimethylsiloxane fiber membranes were fitted [4]. Subsequently, many applications of high-throughput 96-fiber SPME in different geometries (rod fiber, disk and thin film) were studied, utilizing a multifiber device and Concept 96-autosampler. Increasing the surface area of the SPME coating has led to significant improvements in the analytical sensitivity of the assay, even providing exhaustive recovery at equilibrium for some analytes [5]. Recently, an improved portable in vivo multi-sample desorption device, which simplifies the exposure of fiber coatings into all 96 wells, has also been studied [4]. Improvements of high-throughput automated SPME systems have had a significant impact on reducing the time and cost, and increasing the precision and throughput of analysis. A future improvement of automated SPME would be the 384- and 1536-well-plate formats, to further increase sample throughput.

\section{- Sampling devices}

In vivo sampling device for animals \& plants

Solid-phase microextraction devices allow for simultaneous sampling and sample preparation within a living system. The SPME in vivo sampling procedure consists of insertion of the SPME fiber directly into the living system, either to a vein or tissue (e.g., brain, muscle, adipose tissue or liver) of the animal, or a part of a plant (e.g., leaf, stem or bulb). A general tool for direct in vivo SPME is the direct exposure of a biocompatible SPME coating (which is housed inside a hypodermic needle) into the studied matrix, and the subsequent retraction of the coating into the needle for protection and storage. Application of in vivo SPME for pharmacokinetic studies of small animals necessitates an interface to prevent direct insertion of the SPME probe into the animal, due to the smaller blood vessel size. For sampling of large animals, such as a dog, the

SPME probe is sufficiently small to be directly inserted via an indwelling catheter [6,7]. For fish sampling, a needle is used to penetrate the muscle and then removed prior to insertion of the SPME probe into the muscle for sampling [8]. The improvement in in vivo SPME sampling devices for animals has been a milestone in clinical and biological studies. Since no blood/tissue specimen is withdrawn, it provides the ability to study a variety of in vivo equilibria in conscious and freely moving animals. Furthermore, in vivo SPME sampling improves the quality of analytical data by reducing the number of sample handling steps.

"The improvement in in vivo SPME sampling
devices for animals has been a milestone in
clinical and biological studies."

Sampling device for breath \& skin analysis

Breath and skin analysis are non-invasive sampling tools, in which the analysis can be performed without disturbing the biological system of the human or animal. Noninvasive sampling provides the opportunity for more frequent sampling, decreased discomfort and a minimized risk of infection. SPME breath sampling is performed via an SPME fiber protected by inert tubing with a small opening, which allows the patient's breath to pass over the exposed fiber [9]. Human skin sampling can be performed by rolling a commercially available polydimethylsiloxane stir-bar over the specific area of human skin, or placing commercial skin sampling patches covered with cotton wool pads onto the skin, for a specified sampling time $[10,11]$. Breath and skin samples can be used for disease detection and studying metabolomic changes in the body.

\section{- Environmental \& food studies}

Solid-phase microextraction has numerous applications in the area of environmental studies. It can be used for the analysis of a wide range of environmental matrices, including soil, sediment, water and air samples. The development of new sampling devices and calibration methods has accelerated the application of SPME to on-site sampling for both grab sampling and long-term monitoring [12]. Different procedures have been described for quantitative analysis of various volatile and semi-volatile compounds in solid samples, such as soil, sediment, sludge, sand, clay matrices and landfill soils [13]. In addition, aqueous 
sampling by SPME can be performed for the analysis of various compounds in different types of water samples, such as river, sea, rain, waste and drinking water [12-14]. Many applications of SPME have been reported for the analysis of volatile compounds in indoor and outdoor air, gaseous industrial effluents and the lower troposphere [15]. Food analysis is important for the determination of nutritional value, impurities, additives, toxic contaminants and natural ingredients that usually contribute to the particular flavor of the food [13,14]. To date, SPME has been applied to various analyses of aromas, flavors, pesticides, herbicides and fungicides in food samples [16]. As SPME has an open-bed stationary phase, it can directly handle complex food and environmental matrices containing particulate matter and unprocessed samples without the need for sample pretreatment. This also prevents clogging or contamination, which is a frequent limitation in conventional packedbed systems. As a simple, portable, easy-to-use and reliable method, SPME is a suitable tool for field sampling in food and environmental (air, water and soil) studies. The application of SPME with portable analyzer systems to on-site environmental and food analysis allows immediate access to the analytical results, which can then be used to make decisions in the field. In addition, the time interval between sampling and analysis is close to zero, eliminating any possible change in the extracted samples and giving the results in real time.

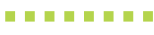 \\ "As SPME has an open-bed stationary phase, it can directly handle complex ... matrices containing particulate matter ... without the need for sample pretreatment. This also prevents clogging or contamination, which is a frequent limitation in conventional packed-bed systems."}

\section{- Clinical toxicology \& forensic analysis}

Solid-phase microextraction is a useful tool in toxicological laboratories, since it can be used for the determination of various compounds with satisfactory sensitivity and precision. Among the many substances that can be analyzed with SPME are narcotics, amphetamines, methamphetamines and related drugs, stimulants, cannabinoids, opioids, illicit drugs and anti-doping substances, as well as drugs that are often a cause of fatal poisoning due to overdose, for example, barbiturates and antidepressants. The substances are extracted either in the headspace or direct immersion mode and can be taken from various matrices such as blood, urine, plasma, serum, tissues, skin or hair. Although the assay is usually selective and compound-specific, there are also methods for more global screening, such as allowing simultaneous detection of many compounds (e.g., 40 narcotics and stimulants [17]), thereby saving time and cost. The simplicity of combining sampling with extraction makes SPME a suitable method for on-site analysis, which can be particularly advantageous in forensic analysis. In addition, it has been shown that many substances can be stored on a fiber for several hours, and so transport of the material from the sampling site to the analytical facility would not be an issue.

\section{- In vivo pharmacokinetics}

In vivo study is a unique feature of SPME when compared with traditional extraction methods. It gives the results reflecting the real condition at the time of sampling, particularly important in the case of drugs and metabolites, which are characterized by low in vitro stability or fast turnover. The first in vivo study on the determination of drug concentrations in a dog was reported in 2003 [18]. Miniaturization of the sampling device then allowed sampling from small animals, such as rodents, enabling the full pharmacokinetic profile of drugs to be obtained [6]. The most advantageous aspect of these studies is the possibility of repeat sampling of the same animal, as this provides more reliable results due to the elimination of inter-individual variability. Furthermore, the number of animals required for a particular experiment is minimized, which significantly decreases the cost of analysis, especially in the case of precious species and lines. In parallel to the development of sampling devices, influential progress in calibration methods has been made. This enables determination of both the free and total concentrations of $\operatorname{drug}(s)$ in the system under study and gives additional information about protein binding. Very promising ongoing studies on the determination of neurotransmitter levels in the brain show that SPME could be an alternative to microdialysis, as SPME causes less damage in living tissue due to the smaller dimensions of the SPME probe. It also allows better spatial and time resolution [19]. A similar approach could be applied to the sampling of other tissues in order to study the local pharmacokinetics and/or metabolomics (tissue response). 


\section{- Metabolomics}

New trends in various areas of science drive the evolution, of analytical techniques. For the past few years, -omics studies have been shown to be a promising diagnostic tool in medicine, where they are used for biomarker discovery and monitoring the progress of therapy. Due to the variability of geometries, sorbents and sampling devices, SPME can be used for targeted and global analysis of volatile, semi- and non-volatile compounds. The most widespread approach in biomarker discovery is the analysis of volatile compounds. Breath, blood and tissue analysis resulted in the discovery of several potential biomarkers of lung, skin and liver cancer in human subjects.

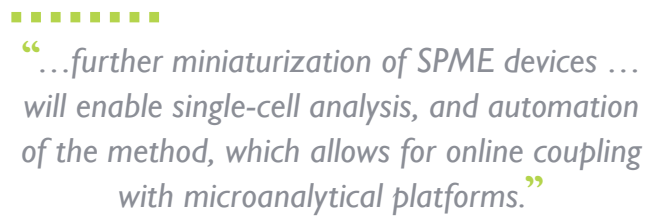

Recent global metabolomics SPME studies were conducted in vivo on mice and the results were compared with those obtained by in vitro DI-SPME, ultrafiltration and protein precipitation. This comparison showed that, among the four techniques used for the studies, only in vivo SPME was capable of capturing fast turnover and unstable compounds [20]. This observation provides new possibilities for the determination of short-lived metabolites, which participate in physiological or pathological pathways that have not been identified to date. Consequently, this would allow for a better understanding of the mechanisms of diseases and the discovery of new biomarkers.

\section{Future directions}

Due to the wide variety of sorbent chemistry and geometries available, as well as the development of sampling devices, SPME has particular potential for use in many sub-specialties of medicine. Automation provides an advantage to hospital laboratories, where a great number of samples require analysis. Biocompatible fibers used for in vivo extraction in laboratory animals, especially rodents, could significantly reduce animal use and decrease the cost of analysis. In the future, in vivo human sampling could be an alternative for traditional blood collection in cases where blood withdrawal is inadvisable, that is, pediatric patients and blood loss or coagulation disorders. One of the most promising features of in vivo SPME is the ability to capture unstable compounds, thus, the potential use of this method for metabolomics studies is not overrated. As mentioned previously, the development of novel techniques is directed by the current trends and needs of science in the widest meaning. Hence, we may expect further miniaturization of SPME devices, which will enable single-cell analysis, and automation of the method, which allows for online coupling with microanalytical platforms.

\section{Financial \& competing interests disclosure}

Janusz Pawliszyn holds the solid-phase microextraction (SPME) patent and the University of Waterloo receives royalties for the sale of SPME devices. Sigma-Aldrich commercializes SPME devices. The authors have no other relevant affiliations or financial involvement with any organization or entity with a financial interest in or financial conflict with the subject matter or materials discussed in the manuscript apart from those disclosed.

No writing assistance was utilized in the production of this manuscript.

\section{Bibliography}

1 Pawliszyn J. SPME commercial devices and fiber coatings. In: Handbook of SPME. Chemical Industry Press, Beijing, China, 86-115 (2009).

2 Ouyang G, Pawliszyn J. A critical review in calibration methods for solid-phase microextraction. Anal. Chim. Acta. 627, 184-197 (2008).

3 Pawliszyn J, Pedersen-Bjergaard S. Analytical microextraction: current status and future tends. J. Chromatogr. Sci. 44, 291-307 (2006).

4 Vuckovic D, Zhang X, Cudjoe E, Pawliszyn J. Solid-phase microextraction in bioanalysis: new devices and directions. J Chromatogr. A 1217, 4041-4060 (2010).

5 Mirnaghi FS, Chen Y, Sidisky LM, Pawliszyn J. Optimization of the coating procedure for a high-throughput 96-blade solid-phase microextraction system coupled with LC-MS/MS for analysis of complex samples. Anal. Chem. 83(15), 6018-6025 (2011).

6 Vuckovic D, de Lannoy I, Gien B et al. In vivo solid-phase microextraction for single rodent pharmacokinetics studies of carbamazepine and carbamazepine-10,11epoxide in mice. J. Chromatogr. A 1218 , 3367-3375 (2011).
7 Musteata FM, Musteata ML, Pawliszyn J. Fast in vivo microextraction: a new tool for clinical analysis, Clin. Chem. 52(4), 708-715 (2006).

8 Zhou SN, Oakes KD, Servos MR, Pawliszyn J. Application of solid-phase microextraction for in vivo laboratory and field sampling of pharmaceuticals in fish. Environ. Sci. Technol. 42, 6073-6079 (2008).

9 Graham AM, Walker V. Headspace solidphase microextraction procedures for gas chromatographic analysis of biological fluids and materials. J. Chromatogr. A 902, 267-287 (2000). 
10 Soini HA, Bruce KE, Klouckova I, Brereton RG, Penn DJ, Novotny MV. In situ surface sampling of biological objects and preconcentration of their volatiles for chromatographic analysis. Anal. Chem. 78, 7161-7168 (2006).

11 Riazanskaia S, Blackburn G, Harker M, Taylorb D, Thomas CLP. The analytical utility of thermally desorbed polydimethylsilicone membranes for in vivo sampling of volatile organic compounds in and on human skin. Analyst 133, 1020-1027 (2008).

12 Ouyang G, Pawliszyn J. SPME in environmental analysis. Anal. Bioanal. Chem. 386, 1059-1073 (2006).

13 Vas G, Vákey K. Solid-phase microextraction: a powerful sample preparation tool prior to mass spectrometric analysis. J. Mass. Spectrom. 39, 233-254 (2004).
14 Risticevic S, Niri VH, Vuckovic D, Pawliszyn J. Recent developments in solidphase microextraction. Anal. Bioanal. Chem. 393, 781-795 (2009).

15 Demeestere K, Dewulf J, De Witte B, Van Langenhove H. Sample preparation for the analysis of volatile organic compounds in air and water matrices. J. Chromatogr. A 1153 , 130-144 (2007).

16 Ridgway K, Lalljie SPD, Smith RM. Sample preparation techniques for the determination of trace residues and contaminants in foods. J. Chromatogr. A 1153, 36-53 (2007).

17 Strano-Rossi S, Molaioni F, Botre F. Application of solid-phase microextraction to antidoping analysis: determination of stimulants, narcotics, and other classes of substances excreted free in urine. J. Anal. Toxicol. 29, 217-222 (2005).
18 Lord H, Grant R, Walles M, Incledon B, Fahie B, Pawliszyn J. Development and evaluation of a solid-phase microextraction probe for in vivo pharmacokinetic studies. J. Anal. Chem. 75, 5103-5115 (2003).

19 Zhang X, Oakes KD, Luong D et al. Temporal resolution of solid-phase microextraction: measurement of real-time concentrations within a dynamic system. Anal. Chem. 82, 9492-9499 (2010).

20 Vuckovic D, de Lannoy I, Gien B et al. In vivo solid-phase microextraction: capturing the elusive portion of metabolome. Angew. Chem. Int. Ed. 50, 5344-5348 (2011).

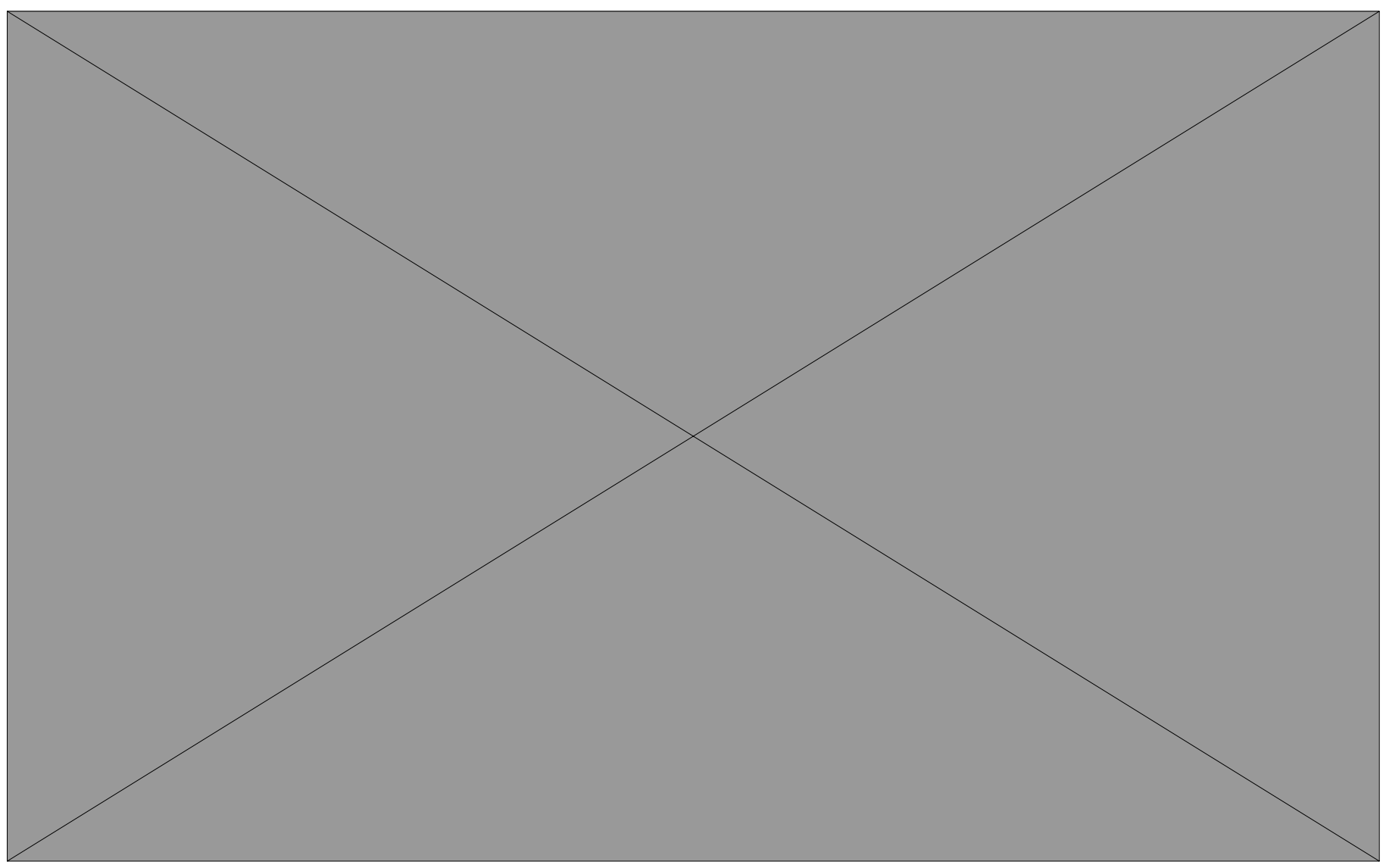

